\title{
Enzimas del metabolismo del etanol: su posible contribución a la predisposición genética del alcoholismo
}

\author{
Sanchis Fortea, M.*; Cuevas Badenes, J.**; Sanchis Arnau, Ma A.*** \\ * Médico Jefe Clínico Unidad Desintoxicación Hospitalaria Bétera (Valencia). \\ * Médico Responsable Unidad Alcohología. Valencia. \\ *** Lcta. Farmacia. Valencia. \\ Enviar correspondencia a: \\ Manuel Sanchis Fortea. Valle de la Ballestera, 32-6-17ª 46015 VALENCIA. Tel. 9634778 19. Email: msanchis@ctv.es
}

\begin{abstract}
Resumen:
Los recientes estudios sobre genética humana sugieren que el abuso de alcohol y el alcoholismo pueden ser hereditarios. Se han localizado los loci génicos y los genes candidatos para identificar a los individuos de alto riesgo, sobre todo en los estudios genéticos referentes a la Alcohol Deshidrogenasa $(A D H)$ y a la Aldehido Deshidrogenasa (ALDH).

Además, una selectiva selección de tales polimorfismos genéticos puede actuar como un factor de protección frente al abuso de alcohol o de la patología asociada, como es el caso de los portadores de alelos ALDH2 "defectuosos", o por el contrario los que poseen el genotipo heterozigoto ALDH2*1/2 presentan mayor riesgo de alcoholismo que los portadores homozigotos ALDH2*1/1. Lo mismo podríamos afirmar sobre el polimorfismo genético del CYP2E1.

Los altos niveles de acetaldehido y la consiguiente reacción "flushing" que se producen tras el consumo de alcohol en los individuos portadores de estos tipos de polimorfismo genético, parecen ser los factores protectores responsables de la resistencia al alcoholismo en estos casos.

Las posibilidades para el control del alcoholismo a través de una intervención genómica están abiertas.
\end{abstract}

Palabras clave: Alcoholismo, Metabolismo, Genética, Enzimas

\section{Summary:}

The recent studies on human genetics suggest the alcohol abuse and the alcoholism can be hereditary.

The genes have been located loci genic and candidates to identify the individuals of high risk, mainly in the referring genetic studies to Alcohol Dehydrogenase $(A D H)$ and Aldehyde Dehydrogenase (ALDH).

In addition, a selective selection of such genetic polymorphisms can act as a factor of protection as opposed to the abuse of alcohol or the associated pathology, as it is the case of the carriers of " defective " alelos ALDH2, or on the contrary those that have the genotype heterozigoto $A L D H 2 * 1 / 2$ present greater risk of alcoholism than homozigotos carriers ALDH2*1/1. The same we could affirm on the genetic polymorphism of the CYP2E1.

The high levels of acetaldehyde and the consequent reaction " flushing " that take place after the alcohol consumption in the carrying individuals of these types of genetic polymorphism, seem to be the protective factors responsible for the resistance to the alcoholism in these cases.

The possibilities for the control of the alcoholism through a genomic intervention are open.

Key words: Alcoholism, Metabolism, Genetics, Enzymes.

\section{INTRODUCCIÓN}

D esde el descubrimiento de la enzima Alcohol Deshidrogenasa, ADH, en la levadura (Negelein, Wulf, 1937), que explica, mediante la síntesis de etanol desde el acetaldehido generado por la oxidación de distintos hidratos de carbono, la fermentación alcohólica por acción de la enzima pivuvato descarboxilaxa ausente en la especie humana (Stryer, 1995), a la preparación cristalina de la primera ADH procedente del hígado de caballo (Bonnichsen, Wassen, 1948), a las recientes investigaciones sobre los estudios de la genética del alcoholismo, Proyecto COGA (Collaborative Study on the Genetics of Alcoholism) (Reich, et al.,1998; Long, et al.,1998) siempre se ha sospechado que el metabolismo del alcohol desempeña un papel crítico en las consecuencias farmacológicas y toxicológicas derivadas del mismo.

La evidencia clínica diaria del desarrollo de patologías asociadas al consumo de etanol solo en algunos bebedores, el "craving" o el estudio de diversos factores que pueden modificar el índice metabólico del etanol (Abdulla, Badawy, 1979), son algunas de las cuestiones que han posibilitado las investigaciones 
que permiten acercarnos al conocimiento del papel que juegan las enzimas metabolizadoras del alcohol en la susceptibilidad, tolerancia metabólica, y variabilidad en las múltiples consecuencias clínicas que se asocian al síndrome de dependencia alcohólica (Rapaka, et al., 1997).

Aceptando el indiscutible papel que en la génesis y desarrollo de la enfermedad alcohólica desempeñan otros factores individuales y sociales, las bases bioquímicas del alcoholismo están centrando en la actualidad gran parte de sus innumerables investigaciones en dos frentes: evidenciar que el estudio genético en este proceso permita desarrollar fundamentos científicos en su tratamiento y establecer los procesos neuronales que predisponen a la conducta adictiva (Topel, 1985; Bower, 1986).

El alcoholismo como afirma el Dr.Gordis, Director del National Institute on Alcohol Abuse and Alcoholism (NIAAA) puede ser "el más complejo de los complejos trastornos", por cuanto el mismo implica gran multiplicidad de genes, estando involucrados, al mismo tiempo, numerosos sistemas de neurotransmisión y de receptores que pueden diferir de unos individuos a otros, lo que puede producir distinta resistencia y vulnerabilidad al alcohol (NIAAA, 1998).

Una de las líneas fundamentales de la moderna alcohología la constituye el estudio del polimorfismo genético asociado a las enzimas metabolizadoras del alcohol en humanos (Wartburg, 1979; Lieber, 1994) que como veremos tiene numerosas implicaciones en el metabolismo y en los efectos del alcohol tanto en individuos normales como en alcohólicos (Lieber, 1996; Kitson, Weiner, 1996; Crabb, Edenberg, 1996).

\section{BREVE RECUERDO DE LAS VÍAS METABÓLICAS DEL ETANOL}

Tras el consumo de alcohol, se produce su paso desde el estómago e intestino hacia el torrente sanguíneo mediante un proceso de difusión simple. La tasa de absorción depende de varios factores tales como: diferencia en los gradientes de concentración entre la mucosa gastrointestinal y la red sanguínea adyacente, el tipo de bebida alcohólica, el efecto de los alimentos, la ingesta de fármacos que afectan a la motilidad gastrointestinal y al flujo sanguíneo, la actividad enzimática en estómago o su inhibición por medicamentos, etc. (Elbel, Schleyer, 1956; Kalant,1979; Bode, 1980).

Desde hace tiempo se conoce que el proceso de metabolismo del alcohol se produce no solo en el hígado sino también en el estómago y en el intestino delgado (el denominado primer paso metabólico) (Mezey, 1985; Gentry et al. 1994), aunque otros investigadores cuestionan el papel que desempeña el estó- mago en esta fase (Lewitt, 1994). No está definido el porcentaje de alcohol eliminado en este primer paso gástrico (Sato, Kimatura, 1996); aunque comparado con el metabolismo hepático, que oxida entre el 8590\% de etanol ingerido (Moser, et al. 1968; Agarwal, 1998) no parece ser importante, y quizás pueda tener relevancia solo a concentraciones bajas de alcohol (Lieber, et al. 1994).

Igualmente, la administración crónica de etanol reduce la actividad gástrica de la ADH (probablemente secundaria a la lesión de la mucosa gástrica) disminuyendo la magnitud de este primer paso metabólico (Julkunen, 1985).

Recientes estudios sugieren que el etanol puede ser metabolizado por las bacterias de la flora intestinal colónica (Salaspuro, 1996; Seitz, et al., 1996). De importancia, como después analizaremos, será su metabolismo por la catalasa en el sistema nervioso central.

Aunque no es objetivo del presente trabajo el análisis detallado del metabolismo del etanol, sí apuntar que el modelo farmacocinético del mismo sigue los cálculos derivados de las ecuaciones de Widmark (Widmark, 1932) así como los procedentes de los nuevos estudios cinéticos del denominado "metabolismo acelerado del etanol" (SIAM) que se produce entre las dos y cuatro horas tras la ingesta de alcohol (Thurmann, R.G., 1989; Crow, Hardman, 1989).

La eliminación por ciertas rutas metabólicas menores (esterificación de ácidos grasos, glucoronoconjugación, etc.) o por otras vías no oxidativas: orina, respiración, sudor es generalmente inferior al 6-10\%, aún en condiciones de sudoración, poliuria o aceleración del ritmo respiratorio (Harger, Forney, 1963; Agarwal, 1998).

En la Fig.1 se exponen las rutas principales de la oxidación del etanol. Según se observa en la misma, tanto la Alcohol Deshidrogenasa (ADH) como la Aldehido Deshidrogenasa (ALDH) transfieren el hidrógeno a un aceptor Nicotinamida Adenina Dinucleótido (NAD), que se convierte en la forma reducida (NADH).

El exceso de equivalentes reductores en el citosol ( donde se localiza la ADH) no se puede oxidar directamente en la mitocondria, porque sus membranas son impermeables al NADH; el proceso de reoxidación mitocondrial del NAD es indirecto. Se lleva a cabo mediante los sistemas lanzadera sustrato, la función de los cuales es llevar los reaccionantes reducidos de las deshidrogenasas desde el citosol a las mitocondrias, oxidarlos y, finalmente, transportarlos ya oxidados al citosol (Abdulla, Badawy, 1979). ( Fig 2 )

La vía microsomal (Citocromo P450lIE1- CYP2E1) se estimula con el aporte etílico prolongado, y puede producir, incluso en ausencia de alcohol cantidades de " agentes oxidantes" (superóxido o peróxido de hidrógeno) que dañan la membrana celular. (Doria, 1997). 


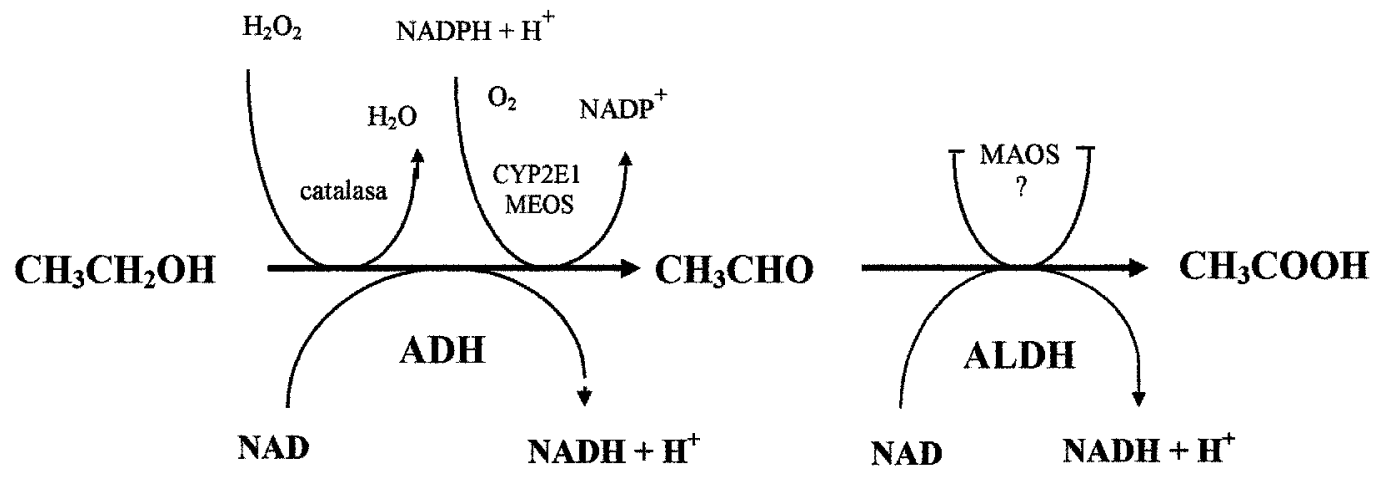

FIGURA 2. VÍAS INDIRECTAS DE OXIDACIÓN DEL NADH
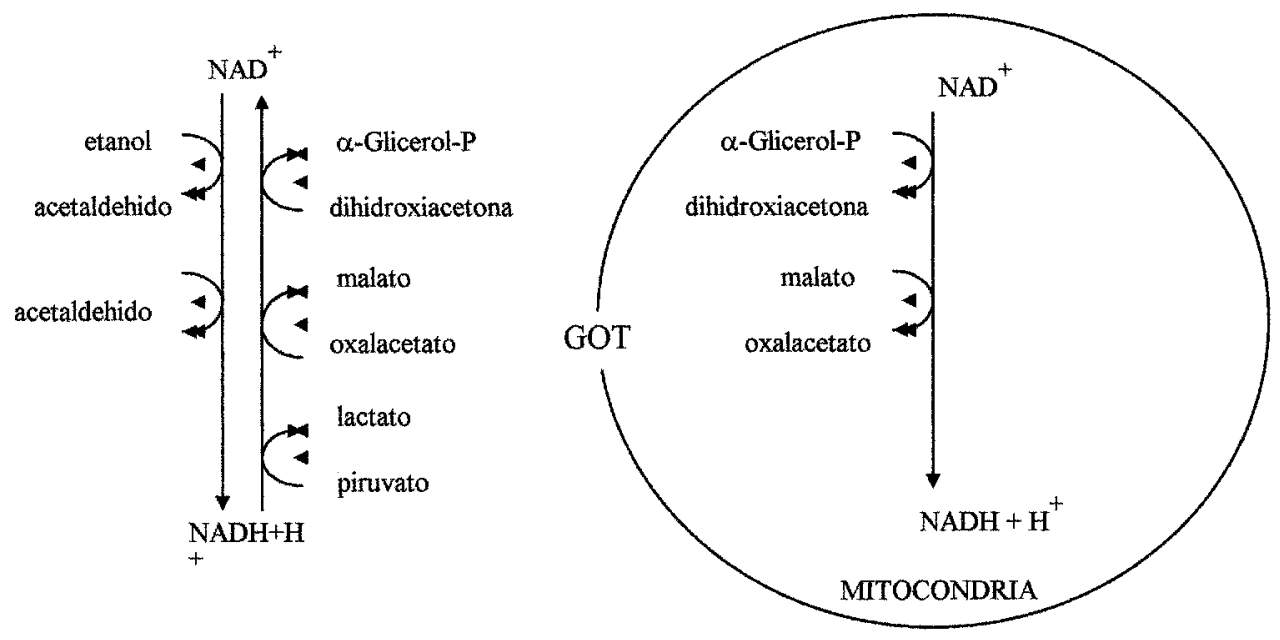

La catalasa en presencia de peróxido de hidrógeno cataliza la oxidación del etanol a acetaldehido.

El acetaldehido producto de las anteriores reacciones catalíticas es un metabolito muy reactivo, siendo considerado responsable de muchas de las acciones tóxicas secundarias al consumo de etanol (Hunt, 1996).

El acetato, resultado final, es metabolizado en otros tejidos, y en el propio hígado, a Acetil-CoA que sigue sus propias rutas metabólicas.

\section{ADH : ALCOHOL DESHIDROGENASA}

La ADH humana hepática es una metaloenzima que consta de dos cadenas polipeptídicas que contie- nen cuatro átomos gramos de Zn por mol de enzima. Los centros activos del enzima uno por cada subunidad contienen dos átomos de $\mathrm{Zn}$, los otros dos restantes probablemente estén relacionados con la estructura terciaria o cuaternaria de la proteina. Asimismo, en cada uno de centros existe un grupo $\mathrm{SH}$ reactivo que interviene en el enlace del etanol y el NAD necesario para la catálisis. (Abdulla, Badawy, 1979; Wartburg, 1979) (Fig. 3)

De localización citosólica, cada subunidad consta de 374 aminoácidos. El número de cambios en los restos aminoácidos, observados en la ADH "clásica" o ADH I, varía en las subunidades alfa, beta y gamma entre 22 y 28 restos, con una secuencia idéntica del 90\% entre todas ellas y un Peso Molecular de 80.000.(Yin, Li, 1989). 


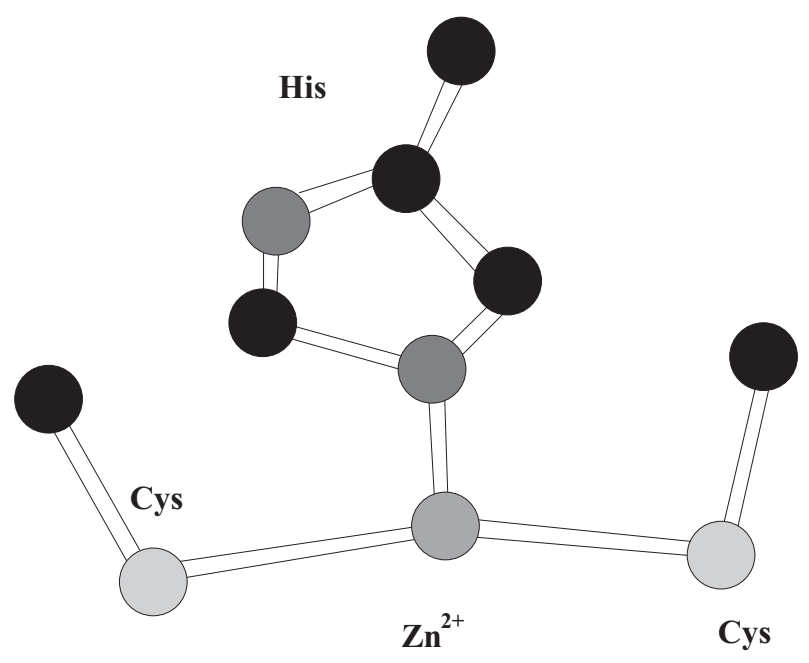

\section{Polimorfismo genético}

La Alcohol Deshidrogenasa, enzima principal del metabolismo del etanol, es en realidad un complejo enzimático en los seres humanos.

De acuerdo con los distintos valores del pH óptimo de acción, con los de la constante de Michaelis-Menten $\mathrm{K}_{\mathrm{m}}$ para el etanol, (cuanto menor es la constante mayor será la afinidad de la enzima para el sustrato) de la $K_{i}$ del inhibidor de la ADH metilpirazol y en función de la identidad secuencial de los aminoácidos que lo conforman, se han identificado, hasta la fecha, seis clases de Alcohol Deshidrogenasa en mamíferos. (Höög, 1998). Todos sus loci génicos están ubicados en el cromosoma 4.

Las deshidrogenasas del alcohol de la clase I (genes $\mathrm{ADH}_{1}, \mathrm{ADH}_{2}$ y $\mathrm{ADH}_{3}$ ), las mejor estudiadas, son las responsábles de la mayor parte del metabolismo del alcohol en los seres humanos. El polimorfismo genético de los genes $A D H_{2}$ y $\mathrm{ADH}_{3}$ da lugar a los diversos alelos que codifican los isoenzimas homo 0 heterodiméricos y que contienen las subunidades alfa, beta y gamma. El alelo $\mathrm{ADH}_{2}{ }^{3}$ que codifica la subunidad beta $_{3}$ posee una actividad 30 veces mayor que la del isoenzima beta beta $_{1}$. Por lo tanto cabe suponer que las personas que lo posean metabolizarán más rápidamente el alcohol (Thomasson et al 1995; Yin, 1994; Xie, 1997; Höög, 1998). (Tabla I)

Las nuevas técnicas de cristalografía por rayos $X$ de alta resolución que analizan la estructura tridimensional de algunos isoenzimas de la clase I evidencian que la sustitución aminoácidos puede afectar la acción catalítica, así por ejemplo el isoenzima beta beta $_{3}$ que difiere del beta $_{2}$ beta $_{2}$, ambos codificados por el gen
$\mathrm{ADH}_{2}$, solo en el resto aminoácido 369, arginina en el priméro y cisteína en el segundo, desestabiliza el complejo NADH-enzima a causa de la arginina y aumenta la constante de disociación para el NAD y NADH (Davis, 1996).

El número de cambios entre la clase I y el resto de clases oscila entre 140 y 180 aminoácidos. (Yin, Li, 1989). No obstante, existen isoenzimas que aunque presenten una gran semejanza en la secuencia de aminoácidos (69\% entre la beta beta $_{1}$ de la ADH I y la sigma sigma de la ADH IV) diferen ampliamente en sus propiedades estructurales y cinéticas (Xie, 1997).

La clase II analizada mediante técnicas de complementariedad de ácido desoxiribonucleico (c-DNA) ha sido determinada en conejos, y caracterizada por dos isoenzimas: el ADH II-1 y ADH II-2 con débil $K_{m}$ para el etanol y con restricciones para alcoholes de más de cinco carbonos (Svensson, 1998). Curiosamente en ratones los isoenzimas de esta clase prácticamente no tienen capacidad de metabolizar el alcohol. (Höög, 1998).

La clase ADH IV es junto con la clase I la más investigada en la actualidad. Está codificada por el gen ADH7 y compuesta por subunidades sigma, estando implicada en el denominado primer paso metabólico del alcohol (Holmes, 1994) siendo su actividad enzimática elevada en estómago y esófago pero no así en el hígado. Asimismo, se ha evidenciado una disminución de su actividad en el sexo femenino. (Carr et al.1996), y al parecer actúa como deshidrogenasa en el metabolismo del retinol. (Satre, et al. 1994; Matsushima, 1995).

Existen numerosas controversias sobre las restantes clases de ADH: La III se detecta en cerebro de 
Tabla I. CLASIFICACIÓN DE LOS ISOENZIMAS DE ADH HUMANA

\begin{tabular}{|c|c|c|c|}
\hline CLASE & GEN & ALELO & SUBUNIDAD \\
\hline I & $\mathrm{ADH}_{1}$ & $\mathrm{ADH}_{1}$ & $\alpha$ \\
\hline \multirow[t]{3}{*}{ I } & $\mathrm{ADH}_{2}$ & $\mathrm{ADH}_{2}{ }^{1}$ & $\beta_{1}$ \\
\hline & & $\mathrm{ADH}_{2}{ }^{2}$ & $\beta_{2}$ \\
\hline & & $\mathrm{ADH}_{2}{ }^{3}$ & $\beta_{3}$ \\
\hline \multirow[t]{2}{*}{ I } & $\mathrm{ADH}_{3}$ & $\mathrm{ADH}_{3}{ }^{1}$ & $\chi_{1}$ \\
\hline & & $\mathrm{ADH}_{3}{ }^{2}$ & $\chi_{2}$ \\
\hline II & $\mathrm{ADH}_{4}$ & $\mathrm{ADH}_{4} ?$ & $\boldsymbol{\pi}$ (en conejos y roedores) \\
\hline III & $\mathrm{ADH}_{5}$ & $\mathrm{ADH}_{5}$ ? & $\begin{array}{l}\text { Formaldehido deshidrogenasa glutation } \\
\text { dependiente (conejos) }\end{array}$ \\
\hline IV & $\mathrm{ADH}_{7}$ & $\mathrm{ADH}_{7}$ & $\sigma$ \\
\hline V & $\mathrm{ADH}_{1} ?$ & $\mathrm{ADH}_{1}(\mathrm{FETAL}) ?$ & \\
\hline VI & $\mathrm{ADH}_{8} ?$ & $? ?$ & Sólo en riñón de roedores \\
\hline
\end{tabular}

ratones (Rout,1992), así como en conejos en los que codifica una formaldehido deshidrogenasa glutation dependiente (Svensson, 1998). Se considera inactiva con el etanol en condiciones fisiológicas (Holmes, 1994).

La ADH V muestra una alta concentración en el hígado fetal humano, indicando que puede ser una forma fetal del isoenzima alfa alfa de la clase I. La clase VI parece ser específica del riñón de roedores (Höög, 1998).

\section{Distribución de la ADH en el organismo huma- no}

El conocimiento de la presencia de actividad catalítica en diferentes órganos es importante por cuanto la posible formación de equivalentes reductores o de acetaldehido puede generar trastornos bioquímicofuncionales en los mismos.
La actividad de la ADH reside, como antes anotábamos, en el citosol celular. Los avances tecnológicos, p.e inmunohistoquímica, análisis secuencial de aminoácidos, cristalografía de rayos $X$, c-DNA complementario desde el m-RNA y PCR, etc., posibilitan nuevos descubrimientos en la ubicación orgánica del sistema ADH.

A modo de ejemplo, exponemos la Tabla II la actividad catalítica de la ADH "clásica", en diferentes órganos humanos (Warburg, 1979).

Por análisis del mRNA para las diferentes clases de Alcohol Deshidrogenasa humana (Engeland, Maret, 1993) encuentran en los tejidos hígado, pulmones, riñón, bazo, estómago, ileon, colon, piel, testículo, útero, la siguiente distribución orgánica:

- Clases I,II y III estaban expresadas en todos los tejidos.

- El m-RNA para la clase I fue el mayor en hígado, pulmón, ileon, colon, y útero

- La mayor expresión para la III era en testículos.

Tabla II

\section{ÓRGANO}

Hígado

Estómago

Intestino

Pulmones

Riñón

\section{OXIDACIÓN ETANOL}

(U. I. por 100 g. de tejido)

\section{ACETALDEHIDO}

805-13150

137

45-87

$128-513$

14-65 
- La clase V no se detectó.

- En estómago se identificó la IV.

Otros estudios encuentran que la clase IV es característica del esófago, que las clases IV y I coexisten en el estómago y que la ADH intestinal es esencialmente de la clase I (Pares, Farres, 1996; Carr, et al., 1996).

La actividad ADH en la flora bacteriana colónica humana y la producción intracolónica de acetaldehido ha sido recientemente investigada en la patogenia de la morbilidad gastrointestinal asociada al consumo de alcohol. La flora aeróbica del colon puede oxidar moderadas cantidades de etanol (Nosova et al.1997; Tillonen et al. 1998). Incluso la producción de origen bacteriano de acetaldehido encontrado en la saliva tras la ingesta de etanol puede ser reducida con antisépticos bucofaríngeeos (Homann, et al., 1998).

\section{Variaciones de los genes de la ADH en diferen- tes poblaciones.}

La relación entre el polimorfismo genético en diferentes poblaciones y razas y el alcoholismo ha sido objeto de numerosos estudios (Yin, Li, 1989; Yin, 1998; Carr, et al.1996; Parés, 1994,1998), etc.

La actividad enzimática de las distintos alelos de la $\mathrm{ADH}$ humana se ha determinado en muestras de distintos tejidos encontrándose que los alelos $\mathrm{ADH}_{2}{ }^{2} \mathrm{y}$ $\mathrm{ADH}_{2}{ }^{3}$ comunes en los orientales pueden metabolizar cuatro veces más etanol $(8 \mathrm{mmoles} / \mathrm{min}$./gramo de tejido) que los fenotipos $\mathrm{ADH}_{2}{ }^{1}$ usuales en la raza europea o caucásica $(2,1 \mathrm{mmoles} / \mathrm{min}$./gramo de tejido), encontrándose, igualmente, que la velocidad máxima en la reacción enzimática del isoenzima beta beta $_{3}\left(\mathrm{ADH}_{2}{ }^{3}\right)$ es treinta veces mayor que la del beta beta ${ }_{1}$ el cual es codificado por la $\mathrm{ADH}_{2}{ }^{1}$ (Yin, Li, . 1989; Thomasson, 1995; Yin, 1998). (Tablas III y IV)

\section{Tabla III. FRECUENCIA DE ALELOS ADH ${ }_{2}$ EN DIFERENTES POBLACIONES}

$\begin{array}{lccc}\text { POBLACIÓN } & \mathbf{A D H}_{\mathbf{2}} \mathbf{1} & \mathbf{A D H}_{\mathbf{2}} \mathbf{2} & \mathbf{A D H}_{\mathbf{2}} \mathbf{}^{\mathbf{N}} \\ \text { CHINOS } & 26 \% & 75 \% & - \\ \text { JAPONESES } & 32 \% & 70 \% & - \\ \text { BRITANICOS } & 95 \% & 4 \% & - \\ \text { ALEMANES } & 95 \% & 4-5 \% & - \\ \text { AMERICANOS } & 80-90 \% & 0-3 \% & 10 \% \\ & & & \text { (Afro-Am.) }\end{array}$

Tabla IV. FRECUENCIA DE ALELOS ADH $\mathrm{ANN}_{3}$ DIFERENTES POBLACIONES

$\begin{array}{lll}\text { POBLACIÓN } & \mathbf{A D H}_{\mathbf{3}} \mathbf{1} & \mathbf{A D H}_{\mathbf{3}} \mathbf{2} \\ \text { CHINOS } & 90 \% & 12 \% \\ \text { JAPONESES } & 88 \% & 13 \% \\ \text { BRITANICOS } & 60 \% & 40 \% \\ \text { ALEMANES } & 60 \% & 42 \% \\ \text { AMERICANOS } & 53 \% & 47 \%\end{array}$

Por lo tanto, puede suponerse que el índice metabólico del etanol, y la consiguiente formación de acetaldehido estaría más acelerado, en estos casos, lo que provocaría, como después analizaremos al estudiar la aldehido deshidrogenasa, una reacción "flushing" que prevendría a los que lo poseen los mencionados coenzimas de un consumo elevado de alcohol.

El actual proyecto COGA, expuesto anteriormente, ha identificado una segunda región en el cromosoma 4, cerca del grupo genético de la $A D H$, sugerente de proteger de la dependencia alcohólica (Long, 1998; Reich,1998). No obstante y por este mismo mecanismo el acúmulo intracelular de acetaldehido sería responsable de un mayor grado de daño tisular y orgánico.

Un reciente estudio multicéntrico efectuado en Europa evidenció que alelo $\mathrm{ADH}_{2}{ }^{2}$ se encontró mas elevado en individuos no alcohólicos (3,8\%) que en pacientes alcohólicos (1,5\%) (Parés, 1998) (Tabla V), al igual que la frecuencia del alelo $\mathrm{ADH}_{2}{ }^{2}$ en razas orientales que se encuentra significativamente disminuida en individuos alcohólicos frente a los del grupo control (Yin, 1998).

\section{Tabla V. FRECUENCIA DE LOS ALELOS DE LA ADH EN PACIENTES ALCOHÓLICOS Y NO CONSUMIDORES*}

\section{RAZA EUROPEA (CAUCÁSICA)}

$\begin{array}{lcc} & \begin{array}{c}\text { ALCOHÓ- } \\ \text { LICOS }\end{array} & \begin{array}{c}\text { NO } \\ \text { ALCOHÓLICOS }\end{array} \\ \text { ALELO ADH }_{2}{ }^{2} & 1,5 \% & 3,8 \% \\ \text { ALELOS ADH } & \text { N.S. } & \text { N.S. } \\ \text { * Asimismo en razas asiáticas (Yin, 1998) } & \end{array}$




\section{CATALASA}

La catalasa, en presencia de peróxido de hidródeno, cataliza la oxidación del etanol a acetaldehido (Keilin, Hartree, 1936, 1945), según la siguiente reacción:

$$
\mathrm{CH}_{3} \mathrm{CH}_{2} \mathrm{OH}+\mathrm{H}_{2} \mathrm{O}_{2} \rightarrow \mathrm{CH}_{3} \mathrm{CHO}+2 \mathrm{H}_{2} \mathrm{O}
$$

De ubicación en los peroxisomas celulares, utiliza el peróxido de hidrógeno generado en las reaccciones catalizadas por enzimas unidas al metabolismo del acetaldehido como son la xantino oxidada y la aldehido oxidasa. (Rajapolan et al. 1964) o por la NADPHoxidasa, de localización microsómica, que oxida al etanol mediante combinación de su propia reacción con la catalasa (Abdulla, Badawy 1979):

$$
\mathrm{NADPH}+\mathrm{H}^{+}+\mathrm{O}_{2} \rightarrow \mathrm{NADP}^{+}+\mathrm{H}_{2} \mathrm{O}_{2}
$$

La presencia de catalasa en el organismo humano, especialmente en hígado, riñón y eritrocitos, junto con la existencia de diversos sistemas generadores de peróxido de hidrógeno podría parecer que desempeñaría in vivo una función activa en el metabolismo del etanol. Los distintos trabajos experimentales no lo han podido comprobar. Así, por ejemplo, la utilización de algunos inhibidores de la catalasa como el aminotriazol se ha demostrado que no altera el índice metábolico de alcohol (Smith, 1961).

Además, en condiciones normales, no es probable que la oxidación del etanol dependiente de la catalasa tenga mucho significado in vivo por el hecho de que el índice de generación de peróxido de hidrógeno en el hígado es escaso en relación al índice metabólico del etanol (Boveris, et al. 1972; Abdulla, Badawy, 1979).

No obstante, se ha sugerido que la catalasa, en condiciones de aporte prolongado de etanol, pudiera estar comprometida en el metabolismo in vivo del alcohol. (Hawkins y Kalant, 1972).

Las investigaciones de los efectos psicofarmacológicos del etanol realizadas en los últimos años, han puesto de manifiesto la presencia de generación de $\mathrm{H}_{2} \mathrm{O}_{2}$ en tejido cerebral de rata y la posibilidad de oxidación del etanol en el cerebro por acción de la catalasa, lo que permitiría comprender el posible papel del acetaldehido en los mecanismos de aversión al consumo de alcohol (Aragon, et al., 1985, 1991; Hunt, 1996).

Por otra parte, no existe una evidencia clara sobre la participación de la ADH o del CYP2E1 en la producción de acetaldehido en los homogenados cerebrales de rata, siendo la fundamentada producción dependiente de la catalasa. Este mismo trabajo pone de manifiesto que tras la ingesta etílica la mayor concen- tración de acetaldehido se localiza en los hemisferios cerebrales y al cabo de 30-60 minutos es máxima en el cerebelo (Liopo, 1998).

En recientes análisis efectuados con homogeneados cerebrales humanos mediante la adición de etanol se ha observado una elevada actividad de la catalasa y un incremento del nivel de acetaldehido en relación con los grupos control (Hamby- Mason, 1997; Hamby- Mason, et al., 1997).

También, algunos de los efectos sobre el Sistema Nervioso Central del alcohol, al menos en animales, pueden ser disminuidos mediante el pretratamiento con el inhibidor de la catalasa el 3- amino 1,2,4 triazol, o aumentados por acción del acetato de plomo, inductor de la misma (Correa, et al., 1998).

\section{EL SISTEMA MICROSOMAL DE OXIDACIÓN DEL ETANOL (MEOS) : CITOCROMO P450IIE1 Ó CYP2E1}

En 1968 Lieber y DeCarli describen un sistema microsómico oxidante del etanol (MEOS) (Lieber, DeCarli, 1968), con capacidad de oxidación del etanol, activo a ph fisiológico y parecido a los enzimas microsomales de desintoxicación de fármacos como la conocida oxigenasa microsómica hepática de función mixta (Conney, 1967). La reacción química producida es:

$$
\begin{aligned}
& \mathrm{CH}_{3} \mathrm{CH}_{2} \mathrm{OH}+\mathrm{NADPH}+\mathrm{H}^{+}+\mathrm{O}_{2} \rightarrow \mathrm{CH}_{3} \mathrm{CHO}+ \\
& \mathrm{NADP}^{+}+2 \mathrm{H}_{2} \mathrm{O}
\end{aligned}
$$

Los hallazgos encontrados por sus descubridores, como son, entre otros, la proliferación del retículo endoplásmico liso, en donde se ubican los microsomas, (Lieber, 1969), tras la administración prolongada de etanol, la posibilidad de inducción por el etanol de sistemas enzimáticos metabolizadores de fármacos (Rubin, et al. 1968) o su implicación en el metabolismo in vivo del etanol han quedado plenamente demostrados.

El sistema MEOS es, en definitiva, un conjunto de enzimas compuesto por un complejo con actividad NADPH oxidasa, por el sistema citocromo P-450 y por el citocromo c reductasa NADPH, con capacidad todos ellos de oxidar diferentes alcoholes. (Teschker, 1975; Lieber, 1977).

Las técnicas de purificación del MEOS mediante utilización de reductasa NADPH citocromo P-450, el uso de fosfolípidos, etc han evidenciado claramente la existencia de una forma específica de citocromo P450 para el metabolismo del etanol (Lieber, 1985).

Se conocen varios tipos de citocromos en animales y en el hombre. De entre los citocromos P450 el 
que es específicamente inducido por el etanol se conoce como citocromo P-450 IIE1 (Lasker,et al.1987; Nebert, et al., 1987) o denominado actualmente CYP2E1. De entre otros citocromos descritos y que puede inducir el etanol como son el CYP3A (Kostrubsky, 1995) o el P450 1A2 solo éste último puede contribuir, con mucha menor proporción, a la oxidación etílica (Asai, et al, 1996).

Aunque este trabajo no pretende estudiar las distintas repercusiones sobre el organismo derivadas del consumo de etanol, si recordar que el CYP2E1 oxida al etanol a acetaldehido fundamentalmente como una monooxigenasa y secundariamente a través de la vía de radicales hidroxilo, con la consiguiente producción de radicales libres, sobre todo en hígado, con el consiguiente estímulo de la peroxidación lipídica y desarrollo de fibrosis hepática. El CYP2E1 tiene capacidad para activar numerosos fármacos con la producción de agentes hepatotóxicos o carcinógenos (Albano, Dianzini, 1996; Lieber, 1997).

De localización en hepatocitos perivenulares en individuos no alcohólicos, se ha demostrado su presencia en pacientes alcohólicos, además, en la zona media y periportal del lobulillo hepático (Cohen, et al. 1997).

El CYP 2E1 ha sido aislado, asimismo, en las células de la mucosa de la lengua y del tracto digestivo alto, incluido e estómago (Shimizu et al., 1990; Seitz, Posch, 1997). Su posible localización cerebral, en fase de experimentación animal, ha evidenciado su presencia en células astrogliales de rata en donde, curiosamente, el Clometiazol, fármaco de amplio uso en la terapia del alcoholismo, inhibe la inducción del mismo (Takada, 1993; Sundberg, Tindberg, 1998).

Con alcoholemias moderadas (alrededor de 60 $\mathrm{mg} / \mathrm{dl}$ ) y mantenidas en el tiempo funciona el sistema MEOS como vía alternativa de la ADH para la oxidación del etanol (Lieber, 1988).

\section{Polimorfismo genético del CYP $2 E 1$}

El polimorfismo genético del CYP 2E1 al igual que se ha demostrado con la ADH y ALDH puede contribuir a explicar las diversas manifestaciones asociadas a la ingesta de bebidas alcohólicas.

Los diferentes estudios analizados sobre el tema, obviamente, de reciente investigación muestran resultados no concluyentes. En este sentido, existen autores que no encuentran diferencias significativas en el genotipo CYP 2E1 entre alcohólicos y no alcohólicos (Nakamura, et al. 1995); no obstante, estos mismos investigadores, en 1996, descubren variaciones en el genotipo CYP 2E1 en voluntarios japoneses tras la inyección intravenosa de etanol: los que poseían el alelo C2 presentaban una concentración alcohólica menor que aquellos que carecían del mismo (Iwahashi, et al, 1996).

Este grupo estudiando el metabolismo del CYP 2E1 hallan diferencias entre los niveles plamáticos de clorzoxazona, sustrato específico del CYP 2E1, entre las muestras procedentes de individuos europeos y japoneses (Kim, et al.1996).

La determinación del polimorfismo genético CYP 2E1 (C/D) por PCR evidenció que la frecuencia del alelo $\mathrm{C}$ en alcohólicos fue significativamente mas elevada que en los controles. Lo que sugiere que el alelo C está asociado a la susceptibilidad del alcoholismo (Ameno, et al. 1998), aunque la frecuencia del alelo C2 en la fibrosis hepática severa o en la cirrosis era menor que en los que no presentaban la referida patología (Agarwall, 1997).

\section{ALDH: ALDEHIDO DESHIDROGENASA}

Como antes afirmábamos el desarrollo de nuevas tecnologías aplicadas al cualquier campo de la ciencia hace posible los numerosos avances producidos en la misma durante los últimos años. $Y$ este es el caso de la genética de las deshidrogenasas ( $A L D H$ ) que oxidan al acetaldehido mediante la transferencia del hidrógeno al NAD tranformándolo en acetato ( Jakobi, 1963):

$$
\mathrm{CH}_{3} \mathrm{CHO}+\mathrm{NAD}^{+}+\mathrm{H}_{2} \mathrm{O} \rightarrow \mathrm{CH}_{3} \mathrm{COOH}+\mathrm{NADH}+\mathrm{H}^{+}
$$

Hasta la fecha se han aislado doce genes que codifican distintos tipos de Aldehido deshidrogenasa en la especie humana enumerados desde la ALDH1 a la ALDH12 (Yoshida, 1998).

Localizados en diferentes cromosomas, Ios loci génicos de las subunidades ALDH1, ALDH2 y ALDH3 están ubicados en los cromosomas 9,12 y 17 respectivamente (Yin, Li, 1989), codificando los distintos grupos de proteinas tetraméricas que oxidan gran variedad de aldehidos alifáticos como el acetaldehido, asi como otro aldehidos de tipo aromático. Entre las distintas ALDHs existe un amplio rango de divergencias en la identidad de sus secuencias aminoácidas (Yoshida, 1998).

\section{Polimorfismo genético}

Al igual que ocurría con la Alcohol Deshidrogenasa, las distintas clases de ALDH están basadas en los valores de $\mathrm{K}_{\mathrm{m}}$ y otras propiedades cinéticas. La clase I de ALDH comprende las isoenzimas ALDH1 con constantes para el acetaldehido $\left(\mathrm{K}_{\mathrm{m}} 30 \mu \mathrm{M}\right)$ y $\mathrm{ALDH} 2$ $\left(K_{m} 2 \mu M\right)$. 
El resto de isoenzimas en función de su elevada constante de Michaelis- Menten para el acetaldehido contribuyen escasamente en el metabolismo del etanol. (Pares, 1996) (Yin, Li, 1989), teniendo una variada distribución en el organismo: tracto gastrointestinal en las isoenzimas 1,2 y 3 (Pares, 1996), glándulas salivares en la ALDH8 (Hsu, 1996).

La ALDH1 y ALDH2 son de localización hepática y están formadas por cuatro cadenas polipeptídicas, con un PM de 54000 en cada subunidad (Craab, 1989). La ALDH1 es la isoenzima citosólica, mientras que la ALDH2 es predominantemente mitocondrial y máxima responsable de la oxidación del acetaldehido.

Existe una identidad secuencial de aminoácidos entre ambas de un $68 \%$. Una variante alélica de la ALDH2, secundaria al cambio en la posición 487 de la cadena polipeptídica del ácido glutámico por la lisina, genera una forma enzimáticamente inactiva.

Tras la administración de una dosis oral de 0,5 grs. de etanol por kilogramo de peso en aquellos individuos que poseen la forma inactiva del isoenzima se encuentran niveles elevados de acetaldehido - $30 \mu \mathrm{M}$ - frente a los - $2 \mu \mathrm{M}$ - hallados en los que presentan la isoenzima ALDH2 activa ( Harada, et al. 1985). Dicha mutación se encuentra en el $50 \%$ de las razas orientales, produciéndose en los mismos una reacción disfórica con "flushing" tras la ingesta de etanol (Wall, 1991).

Estudios posteriores relacionados con los genotipos de la ALDH2 en las razas orientales en pacientes alcohólicos y en los que presentan una reacción "flushing" evidencian una frecuencia elevada en alcohólicos del alelo ALDH2*1/2 ( heterozigoto) (Yin, 1998) los cuales soportan mejor la desagradable reacción que se provoca tras una nueva dosis de etanol ( Fukunaga, 1998). La probabilidad de encontrarnos un alcohólico portador del alelo homozigótico ALDH2*1/1 es menor que en el caso anterior ( Nakamura, et al.,1996); incrementando, por tanto, el riesgo de alcoholismo en los ALDH2*1/2 (Wall, 1998).

Siguen todavía oscuras las razones por las cuales unos pacientes alcohólicos desarrollan ciertas complicaciones órganicas y otros las presentan con menor intensidad o incluso nulas, sin embargo se ha demostrado que la frecuencia del alelo ALDH2 ${ }^{*} 1 / 2$ en los pacientes con cirrosis hepática alcohólica y con pancreatitis aguda era más elevada que en los controles no alcohólicos( Chao, et al., 1997). El consumo crónico de etanol, salvo cuando se acompaña de grave deterioro hepático, no deprime la actividad de la ALDH ( Vidal, F. et al., 1998).

\section{A MODO DE CONCLUSIÓN}

Los recientes estudios sobre genética humana sugieren que el abuso de alcohol y el alcoholismo pueden ser hereditarios. Se han localizado los loci génicos y los genes candidatos para identificar a los individuos de alto riesgo, sobre todo en los estudios genéticos referentes a la Alcohol (ADH) y a la Aldehido Deshidrogenasa (ALDH).

Además, una selectiva selección de tales polimorfismos genéticos puede actuar como un factor de protección frente al abuso de alcohol o de la patología asociada, como es el caso de los portadores de alelos ALDH2 "defectuosos", o por el contrario los que poseen el genotipo heterozigoto ALDH2*1/2 presentan mayor riesgo de alcoholismo que los portadores homozigotos ALDH2*1/1 ( Agarwall, 1997). Lo mismo podríamos afirmar sobre el polimorfismo genético del CYP2E1 ( ver anteriormente).

Las posibilidades para el control del alcoholismo a través de una intervención genómica están abiertas (Chrostek, Szmitkowski, 1998).

\section{REFERENCIAS BIBLIOGRÁFICAS}

Abdulla, A.; Badawy, B. (1979): "El metabolismo del alcohol". En: Marks, Wright, Directs. Clínica Endocrinológica: Efectos Metabólicos del Alcohol. Salvat Editores. Barcelona. pp:1-24.

Agarwal, D.P.(1997): "Molecular genetic aspects of alcohol metabolism and alcoholism". Pharmacopsych., 30, 7984.

Agarwal, D.P.(1998): "Pharmacokinetic and pharmacogenetic aspects of alcohol." In: Snel, Lorist, Eds., Nicotine, Caffeine and Social Drinking. The Nederlands: Harwood. pp:259-274.

Albano, E.; Dianzini, M.U. (1996): "Role of free radicals in alcohol-induced liver damage." Alcologia, 8, 79-84.

Ameno, N. et al. (1998): "DRAl site polymorphism of the human CYP2E1 (Cytochrome P4502E1) gene and alcoholism." $9^{\text {th }}$ ISBRA CONGRESS. Copenhagen, Denmark.

Aragon, C.M.G.; Spivak, K.; Amit, Z. (1985): "Blockage of ethanol induced condicioned taste aversion by 3amino, 1,2,4 triazole: Evidence for catalase mediated synthesis of acethaldehyde in rat brain" Life Sciences, $37,2077-2084$.

Aragon, C.M.G.; Stoland, L.M.; Amit, Z. (1991): “Studies on ethanol-brain catalase interaction: Evidence for central ethanol oxidation." Alcoholism: Clin. Exp. Res., 15, 165169.

Asai, H. et al. (1996): "Microsomal ethanol oxidizing system activity by human hepatic cytochrome P450s." J. Pharmac. Exper. Therap., 277, 1004-1009.

Bode, J. C.(1980): "Alcohol and the gastrointestinal tract" Advanc. Intern. Med. Pediat.,45, 1-75.

Bonnichsen, R.K.; Wassen, A.M. (1948): "Crystalline alcohol dehydrogenase from horse liver". Arch. Biochem. Biophys., 18, 361. 
Boveris, A.G. et al. (1972): "The cellular production of hydrogen peroxide" Biochem. J., 128, 617-630.

Bower B. (1986): "Researchers tackle the mystery of alcoholism." New Physician, 35, 26-28, 42-43.

Carr, L.G. et al.(1996): "Failure to find exon 7 polymorphism in the ADH7 gene in Chinese, Japanese, African-Americans, and Caucasians." Alcoholism: Clin. Exper. Res., 20, 418-419.

Chao, Y.C. (1997): " Alcoholism and alcoholic organ damage and genetic polymorphisms of alcohol metabolizing enzymes in Chinese patients." Hepatology, 25, 112-117.

Chrostek, L.,Szmitkowski, M.(1998): " Genetic evaluation of tolerance to alcohol". Postepy Hig. Med. Dosw., 52, 3547.

Cohen, P.A. et al. (1997): “Immunohistochemical determination of hepatic cytochrome P-4502E1 in formalin-fixed, paraffin-embedded sections." Alcoholism: Clin. Exper. Res., 21, 1052-1062.

Conney, A.H.(1967): "Pharmacological implications of microsomal enzyme induction." Pharmac. Reviews, 19, 317366.

Correa, M. et al. (1998): "Acute lead administration potentiates ethanol- induced locomotor activity: the involvement of brain catalase." $9^{\text {th }}$ ISBRA CONGRESS. Copenhagen, Denmark.

Correa, M. et al. (1998): "Lead acetate and 3-amino-1,2,4triazole on brain catalase and ethanol- induced locomotor activity". $9^{\text {th }}$ ISBRA CONGRESS.Copenhagen, Denmark.

Craab, D.W. et al. (1989): "Genotypes for aldehyde dehydrogenase deficiency and alcohol consumption." J. Clin. Invest., 83, 314-316.

Crabb, D.W.; Edemberg, H.J. (1996): "Gene regulation of alcohol metabolizing enzymes." Suppl. Alcoholism: Clin Exper. Research, 20, 113-116.

Crow, K.E.; Hardman, M.J. (1989): “In: Crow, Batt Eds.: Human Metabolism of Alcohol" Boca Raton, FL:CRC Press.pp. 3-16.

Davis, G.J. et al. (1996): "X-ray estructure of human beta 3 beta 3 alcohol dehydrogenase". J. Biol. Chem., 271, 17057-17061

Doria, J.J. (1997): "Alcohol metabolism". Alcohol Health \& Research World, 21, 323.

Elbel,H., Schleyer, F. (1956): "Blutalkohol. Die Wissenschaftlichen Grundlagen der Beurteilung von Blutalkoholbefinden." 2 Ed. Thieme,G., Stuttgart.

Engeland, K.; Maret, W. (1993): “Extrahepatic differential expression of four classes of human alcohol dehydrogenase". Biochem. Bioph. Res. Commun., 193, 47-53.

Fukunaga, T. (1998): " Evaluation of m-RNA levels for ALDH2 in human peripheral blood before ans after ethanol intake". $9^{\text {th }}$ ISBRA CONGRESS. Copenhagen, Denmark.

Gentry, R.T.; Baraona, E.; Lieber, C.S. (1994): “Agonist: The case againts first pass metabolism of the ethanol in the stomach." J.Labor. Clin. Med., 123, 21-26.

Hamby-Mason, R.L. et al. (1997): "Catalase mediates acetaldehyde formation from ethanol in fetal and neonatal rat brain." Alcoholism: Clin. Exp. Res.,21, 1063-1072.
Hamby-Mason, R.L. (1997): “Catalase-mediated production of acetaldehyde as a mechanism underlying ethanolenhanced apoptosis in the developing brain." Disser. Abs. Intern., 58, 1230-B.

Harada, S. et al. (1985): Alcohol, 2, 391-392.

Harger, R.N., Forney, R.B. (1963): In: Stolman Ed.: Progress in Chemical Toxicology. Academic Press, N. York. Vol 1 p.79.

Hawkins, R.D.; Kalant, H. (1972): "The metabolism of ethanol and its metabolic effects." Pharmacol. Reviews, 24, 67-167.

Holmes, R.S. (1994): "Alcohol dehydrogenases: a family of iszymes with differential functions". Alcohol Alcoholism Suppl. 2, 127-130.

Homann N. Et al. (1998): "Acetaldehyde in saliva: formation and pathogenic aspects" 9th ISBRA CONGRESS. Copenhagen, Denmark.

Höög, J.O. et al (1998): "Structure and function of novel forms of mammalian alcohol dehydrogenase." $9^{\text {th }}$ ISBRA CONGRESS. Copenhagen, Denmark.

Hsu, L.C.; Chang, W:C.(1996): "Sequencial and expression of the human ALDH8 encoding a new member of the aldehyde dehydrogenase family." Gene, 174, 319-322.

Hunt, W.A, (1996): "Role of acetaldehyde in the actions of ethanol on the brain: A review." Alcohol: An Inter. Biochem. J., 13, 147-151.

Iwahashi, K. et al. (1996): "CYP2E1, ALDH2 and ADH2 genotypes and blood ethanol elimination kinetics." Clin. Chim. Acta, 255, 85-87

Jacoby, W.B. (1963): In: Boyer, Lardy, Myrbäck, Eds., In the Enzymes. Academic Press, Inc., New York, pp: 203-211

Julkunen, R.J.K. et al.(1985): “First pass metabolism of ethanol: An important determinant of blood levels after alcohol consumption." Alcohol, 2, 437-441.

Kalant, H. (1979): "Absortion, Diffusion, Distribution, and Elimination ot Ethanol: Effects on Biological Membranes." In: Kissin and Begleiter, Eds. The Biology of Alcoholism. Plenum Press. New York. vol 1 pp.1-62.

Keilin, D.; Hartree, E.F. (1936): "Coupled oxidation of alcohol." Proceed. Roy. Society, 119, 141-159.

Keilin, D.; Hartree, E.F. (1945): "Properties of catalase. Catalysis of coupled oxidation of alcools." Biochem. J., 39, 293-301.

Kim, R.B. et al. (1996): "In vivo and in vitro characterization of CYP2E1 activity in Japanese and Caucasians." J. Pharm. Exper. Ther., 279, 4-11.

Kitson, K.E.; Weiner, H. (1996): "Ethanol and acetaldehyde metabolism: Past, present, and future". Suppl. Alcohol: Clin. Exper. Res., 20, 82A-92A.

Kostrubsky, V. E. et al. (1995): "Ethanol and isopentanol increase CYP3A and CYP2E in primary cultures of human hepatocytes." Archiv.Biochem. Biophys.,322, 516-520.

Lasker, J.M.et al. (1987): "Purification characterization of human liver citocrome P-450-ALC." Biochem. Biophys. Res. Commun., 148, 232-238. 
Levitt, M.D. (1994): "Antagonist: The case againts first pass metabolism of the ethanol in the stomach." J.Labor. Clin. Med., 123, 28-31.

Lieber, C. S. et al. (1996): "First pass metabolism of ethanol." In: Saunders, Whitfield Eds. Biology of Alcohol Problems. Seventh ISBRA Congress. Australia. Pergamon Press. New York. pp: 163-169.

Lieber, C. S., DeCarli L.M. (1968): "Hepatic microsomes: a new site for ethanol oxidation." J. Clin. Invest., 47, 62.

Lieber, C.S. (1994): "Metabolic consequences of ethanol" Endocrinologist, 4, 127-139

Lieber, C.S. (1996): "Metabolism of alcohol and its implications for the pathogenesis of disease". In: Preedy, Watson Eds.: Alcohol and the Gastrointestinal Tract. Boca Raton, FL:CRC Press. pp. 19-29.

Lieber, C.S. (1997): "Cytochrome P-4502E1: Its physiological and pathological role." Physiolog. Reviews, 77, 517-544.

Lieber, C.S. (1998): "Biochemical and molecular basis of alcohol induced injured to liver and other tissues." $N$. Eng. J. Med., 319, 1630-1650.

Lieber, C.S.(1977): Metabolic aspects of alcoholism. MTP, pp: $1-30$.

Lieber, C.S. (1969): “In: Bittar, Ed., “The Biological Basis of Medicine." Academic Press. London. pp:317-344.

Lieber, C.S. (1988): "Biochemical and molecular basis of alcohol indyced injured to liver and other tissues." $N$. Eng.J. Med., 319, 1630-1651.

Liopo, A.V. (1998): "Ethanol metabolism in rat brain: kinetics and regional distribution." $9^{\text {th }}$ ISBRA CONGRESS. Copenhagen, Denmark.

Long J.C. et al (1998): "Neurogenetics Lab scan suggest genetic linkage on chromosomes 4 and 11 ". Neuropsych. Genetics, 81, 3.

Matsushima, T. (1995): "ADH isoenzymes". Nippon Rinsho, 53, 1237-1240.

Mezey, E. (1985): "Effect of ethanol on intestinal morphology, metabolism and function." In: Seitz, Kommerell, Eds., Alcohol related diseases in gastroenterology. Berlin: Springer-Verlag.pp 342-360.

Moser et al (1968): "Heterogenität und Organverteilung der Alkoholdehydrogenase bei verschiedenen Spezies." Enzym. Biol. Clin., 9, 447.

Nakamura, K. et al. (1995): "Association between alcoholics and the genotypes of ALDH2, ADH2, ADH3 as well as P-4502E1." Japan. J. Stud. Drug. Depend., 30, 33-42.

NATIONAL INSTITUTE ON ALCOHOL ABUSE AND ALCOHOLISM (1997): "Alcohol Metabolism.." Alcohol Alert, $\mathrm{N}^{\circ} 35, \mathrm{PH} 371$.

NATIONAL INSTITUTE ON ALCOHOL ABUSE AND ALCOHOLISM (1998): Press: 301/443-3860.

Nebert, D.W. et al. (1987): "The P-450 gene superfamily, recommended nomenclature." DNA, 6, 1-8.

Negelein, E., Wulff, H.J.(1937): “Diphosphopyridinproteid, Alkohol, Acetaldehyd." Biochem.Z.293:351.

Nosova, T. et al. (1997): "Characteristics of alcohol dehydrogenases of certain aerobic bacteria representing human colonic flora". Alcoholism: Clin. Exp. Res.,21, 489-494.
Parés, X. et al (1994): "Genetic polymorphism of liver alcohol dehydrogenase in Spanish subjects: significance of alcohol consumption and liver disease." Alcohol Alcohol., 29, 701-705.

Parés, X. et al.(1998): "Alcohol Dehydrogenase genotypes in Europeans". 9'th ISBRA CONGRESS.Copenhagen, Denmark.

Pares, X.; Farres, J. (1996): "Alcohol and aldehyde dehydrogenases in intestinal tract." In: Preedy, Watson, Eds., Alcohol and the Gastrointestinal Tract. Boca Raton, CRC Press.pp: 41-46.

Rajagopalan, K.V.; Handler, P. (1964): "Hepatic aldehyde oxidase." J. Biol. Chem., 239, 2022-2027.

Rapaka R.S. et al. Eds. (1997): Pharmacokinetics, metabolism and pharmaceutics of drug of abuse. NIDA Research Monograph $n^{\circ}$ 173. Rockville, MD: NIDA.

Reich, T et al.(1998): "COGA genome scan suggest linkage on chromosomes 1,2,4 and 7" Neuropsych. Genetics, $81,3$.

Rout, U.K. (1992): "Alcohol dehydrogenases in the brain of mice." Alcoh. Exper. Research, 16, 286-289.

Rubin, E. et al. (1968): “Ethanol increases hepatic smooth endoplasmic reticulum and drug-metabolizing enzymes." Science, 159, 1469

Salaspuro, M. (1996): “Bacteriocolonic pathway for ethanol oxidation: Characteristics and implications." Annals of Medicine, 28, 195-200.

Sate, M.A. et al. (1994): "Complete structure of human class IV alcohol dehydrogenase(retinol dehydrogenase) determined from the ADH7 gene." J. Biol. Chem., 269, 15606-15612.

Sato, N., Kitamura, T. (1996): "First-pass metabolism of ethanol: An overview" Gastroenterology, 111, 1143-1150.

Seitz, H.K. et al. (1996): "Ethanol metaolism in the gastrointestinal tract and its possible consequences." In: Saunders and Whitfield Eds., Biology of Alcohol Problems. $7^{\text {th }}$ ISBRA Congress. Australia. Pergamon Press. New York. pp 157-162.

Seitz, H.K.; Poschl, G. (1997): "Role of gastrointestinal factors in alcohol metabolism." Alc.Alcoholism: Int. Med. Council Alcoh., 32, 543-549.

Shimizu M. et al. (1990): "Inmunohistochemical of localization of ethanol inducible P450IIE1; alimentary tract." Gastroenterology, 99, 1044-1053.

Smith, M. E. (1961): "Interrrelations in alcohol and methanol metabolism." J. Pharm. Exp. Ther., 134, 233-237.

Stryer, L (1995): Bioquímica. $4^{a}$ Ed. Reverté, S.A. Barcelona.

Sundberg, M.I.; Tindberg, N. (1998): "Mechanisms of induction of cytochrome P4502E1 in astroglial cells." $9^{\text {th }}$ ISBRA CONGRESS.Copenhagen, Denmark.

Svensson, S. et al. (1998): "Structural and functional divergence of class II alcohol dehydrogenase: Cloning and characterisation of rabbit liver isoforms of the enzyme." Europ. J. Biochem., 252, 236-243.

Takada, A. Ed. (1993): "Proceedings of the $13^{\text {th }}$ annual conference of the Japanese Society for Biochemical Research on Alcohol. Osaka. Oxford, UK: Pergamon Press. 
Teschker et al. (1975): "Hepatic microsmal alcohol oxidazing system: affinity for methanol, ethanol, propanol and butanol." J. Biol. Chem., 250, 7397.

Thomasson H.R. et al. (1995): "ADH2 gene polymorphism are determinants of alcohol pharmacokinetics." Alcohol. Clin. Exp. Res., 19,1494-1499.

Thurman, R.G. et al. (1989): "Swift increase in alcohol metabolism (SIAM): A commentary on the regulation of alcohol metabolism in mamals." In: Crow, Batt Eds., Human Metabolism of Alcohol. Boca Raton, FL:CRC Press.pp. 17-30.

Tillonen, J. et al. (1998): "Effects of ciprofloxacin on bacteriocolonic pathway for ethanol oxidation in man". $9^{\text {th }}$ ISBRA CONGRESS.Copenhagen, Denmark.

Topel, H. (1985): "Biochemical basis of alcoholism: Statements and hypotheses of present research." Alcohol, 2, 711-788.

Vidal, F. et al. (1998): "Influence of chronic alcohol abuse and liver disease on hepatic aldehyde dehydrogenase activity". Alcohol, 15, 3-8.

Wall, T.L. (1991): "Electroencephalographic correlates of alcohol-induced flushing in Asian-American men". Dissert. Abstr. Internat., 52, 2789-B.

Wall, T.L. et al. (1998): " Self-rating of alcohol's effects in Asians with aldehyde dehydrogenase (ALDH2) gene mutations." $9^{\text {th }}$ ISBRA CONGRESS.Copenhagen, Denmark.

Wartburg, von J.P. (1979): "The Metabolism of Alcohol in Normals and Alcoholics: Enzymes" In: Kissin and Begleiter, Eds., The Biology of Alcoholism. Plenum Press. New York. vol 1 pp.63-101

Widmark, E.M.P. (1932): Die theoretischen Grundlagen und die praktische Verwendbarkeit der gerichlich - medizinischen Alkoholbestimmung. Berlin: Urban, Schawarzenberg.

Xie, P. et al. (1997): "X-ray structure of human class IV sigma sigma alcohol dehydrogenase: Structural basis for substrate specificity." J. Biol. Chem., 272, 18558-18563

Yin, S-J., Li T-K. (1989): "Genetic polymorphism and properties of human alcohol and aldehyde dehydrogenases: implications for ethanol metabolism and toxicity." In: Sun G.Y.et al. Eds., Molecular Mechanisms of Alcohol. Humana Press. Inc.New Jersey

Yin, S. J. (1998): "Allelic variations of the Alcohol- Dehydrogenases and alcoholism in Asians". 9 $9^{\text {th }}$ ISBRA CONGRESS. Copenhagen, Denmark.

Yin, S.J. (1994): "Alcohol dehydrogenase: Enzymology and Metabolism." Alcohol Suppl. 2, 113-119

Yoshida A. et al. (1998): "Human aldehyde dehydrogenase gene family" Eur. J. Biochem., 251, 549-557. 